\title{
Influência da divisão do céu em cálculos de disponibilidade de luz a partir de imagens de grande alcance dinâmico
}

\author{
SOUZA, Dennis F. ${ }^{1}$ \\ SCARAZZATO, Paulo S. ${ }^{2}$ \\ PEDRINI, Hélio \\ ${ }^{1}$ Universidade Estadual de Campinas, Campinas, Brasil. dennis.souza@gmail.com \\ ${ }^{2}$ Universidade de São Paulo e Universidade Estadual de Campinas, São Paulo, Brasil. pasezato@usp.br
}

\begin{abstract}
Resumo
Os sistemas digitais cumprem um importante papel em estudos que envolvem a medição de céu, em virtude de suas possibilidades de análise. Atualmente, os equipamentos disponíveis para o registro de luminâncias são programados a partir de divisões de céu recomendadas pela Comissão Internacional de lluminação (em francês, Commission Internationale de L'éclairage - CIE), válidas para céus encobertos, mas com a presença do sol tais valores podem ser equivocadamente interpretados.Por sua vez, as imagens digitais de Grande Alcance Dinâmico(em inglês,High Dynamic Range- HDR) não têm essa limitação e podem ser subdivididas em qualquer número de seções ou zonas. Este artigo avalia a influência da divisão do céu em cálculos de luminância e iluminância. Os valores obtidos a partir das imagens HDR foram divididos com o uso de diversos métodos de segmentação, e os cálculos foram comparados a medições feitas com luxímetros. Os resultados sugerem que maiores subdivisões conseguem lidar melhor com a presença do sol e podem ser úteis para o registro apropriado de condições de céu claro e parcialmente nublado.
\end{abstract}

Palavras-Chave: iluminação natural, imagens HDR, medição, comparação estatística.

\begin{abstract}
Digital systems play an important role in sky measurements due to the evaluation possibilities they possess. Current equipments used for sky vault luminance measurements are programmed according to the International Commission on Illumination (in french, Commission Internationale de L'éclairage - CIE) recommended sky division, useful for overcast skies, but when the sun is present the luminance values may be incorrectlyinterpreted. High Dynamic Range (HDR) images do not show this kind of limitation and can be divided into any number of segments and zones. This paper investigates the influence of sky subdivision in luminance and illuminance calculations. The HDR values were divided using several segmentation methods, and calculations were compared to measurements made with illuminance meters. The results suggest that larger subdivisions deal better with the solar corona and may be useful to properly register the partly cloudy and clear sky conditions.
\end{abstract}

Keywords: daylight, HDR, measurement, statistical comparison. 


\section{Introdução}

Sistemas digitais vêm sendo cada vez mais empregados em estudos de iluminação no ambiente construído, dada sua versatilidade e possibilidades de uso. No campo da luz natural, é comum o uso de imagens da abóbada celeste (ROY et al., 1998; CHAMAIDI, 2006; PEREIRA, 2008).Frequentemente, esta forma de registro esteve restrita à caracterização do tipo de céu e à análise visual das condições de iluminação, devido às limitações inerentes ao processo de registro da imagem. Os valores de luminâncias na abóbada celeste são geralmente fornecidos por equipamentos de medição pontual, como os luminancímetros e os sky scanners utilizados em estações medidoras, construídos a partir de divisões do céu recomendadas pela Comissão Internacional de lluminação (em francês,Commission Internationale de L'éclairage - CIE). Tal modelo, proposto por Tregenza (1987), é válido para céus encobertos, mas a presença do sol pode fazer com que os valores obtidos sejam equivocadamente interpretados.

O desenvolvimento recente das técnicas, em especial com as imagens de Grande Alcance Dinâmico (eminglês, High Dynamic Range - HDR), possibilitou o registro da distribuição de luminâncias, recurso inimaginável quando consideradas as limitações originais das câmeras fotográficas, que utilizam por padrão imagens de Baixo Alcance Dinâmico (em inglês,Low Dynamic Range - LDR). Inicialmente, os estudos com HDR apontavam para as dificuldades inerentes ao processo de captura de cenas com alto contraste, como o registro de céus claros (STUMPFEL et al., 2004; REINHARD et al., 2006). Novas pesquisas, contudo, atestam a possibilidade de tais registros, que podem ser utilizados em novos métodos de simulação virtual (CHENEY; INANICI, 2008; INANICI, 2009), construídos sob o princípio de renderização baseada em imagens (em inglês, Image-Based Rendering - IBR). Para programas que funcionam a partir da renderização baseada no comportamento físico real da luz (em inglês, Physically-Based Rendering - PBR), os modelos preditivos ainda são a única fonte de luz natural disponível para a realização das simulações.

\section{Objetivo}

O objetivo deste artigo é avaliar a influência dos métodos de divisão do céu nos cálculos de distribuição da luz, a partir da medição da abóbada celeste com o uso de imagens HDR.

\section{Método}

O método deste trabalho está dividido em três partes:

Registro da abóbada celeste.

Extração dos dados em diversos números de divisões de céu.

Comparação entre valores calculados e medição por luxímetro.

\subsection{Registro da abóbada celeste}

O registro da distribuição da luz no céu foi realizado a partir da calibração dos equipamentos de medição e da obtenção das imagens HDR, utilizando-se os equipamentos e programas descritos na Tabela 1. Ambas as etapas valeram-se de um sistema de controle remoto por meio de conexão USB entre a câmera e o computador, de forma a assegurar o mínimo de oscilação possível devido à intervenção do usuário, minimizando possíveis erros de enquadramento e alinhamento das imagens.

Tabela 1: Equipamentos utilizados no registro da abóbada celeste.

\begin{tabular}{|l|l|}
\hline Câmeras Fotográficas & Nikon D60 e Canon EOS Rebel XSi \\
\hline Objetivas & $\begin{array}{l}\text { 2x Sigma 4.5mm f2.8/22 180 - circular fisheye } \\
(1 \mathrm{x} \text { Nikon mount e 1x Canon mount) }\end{array}$ \\
\hline Computador & Macbook Air modelo MC966BZ/A \\
\hline Luminancímetro & Minolta LS-110 \\
\hline Luxímetro & Minolta T-10 \\
\hline Controle das câmeras & Icarus Camera Control s20100408 \\
\hline Geração de Imagens HDR & Photosphere 1.8.7U \\
\hline Programação & MATLAB 2011b \\
\hline
\end{tabular}

\subsubsection{Calibração dos equipamentos}

O luminancímetro e o luxímetro já se encontravam calibrados para o uso na pesquisa.As câmeras fotográficasforam calibradas de acordo com métodos já utilizados em pesquisas anteriores e já consagrados por outros estudos (INANICI; GALVIN, 2004; INANICI, 2005 e JACOBS, 2007). Essa calibração foi realizada por meio da medição de um quadro em tons de cinza em 11 pontos (Figura 1), com as câmeras e o luminancímetro. Com isso, foi possível obter as Curvas de Resposta (CR) e os Fatores de Calibração (FC).

A CR é obtida por meio da análise pontoaponto da HDR de referência, criando-se uma curva polinomial que pode ser utilizada em outras séries de imagens. Neste estudo, recorreu-seao modelo de Mitsunaga e Nayar (REINHARD et al., 2006), algoritmo-base do programa Photosphere(WARD, 2012). O FC é uma unidade escalar que estabelece uma relação entre os valores obtidos com um equipamento calibrado, como 
Figura 1: Quadro de Calibração.

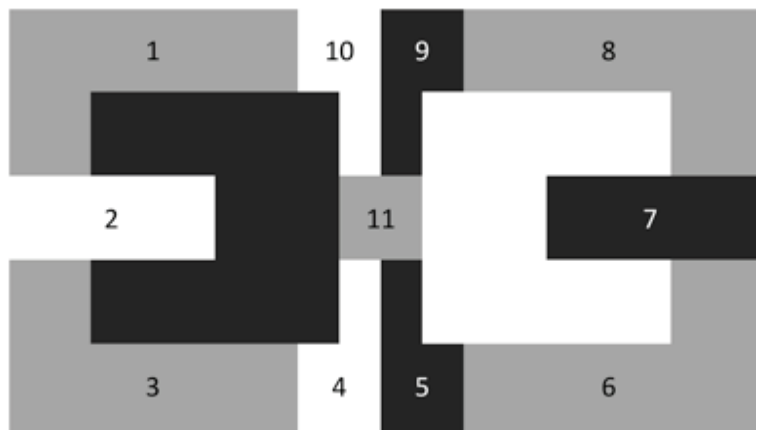

o luminancímetro, e os valores obtidos a partir da imagem HDR. Esses dois parâmetros são necessários para assegurar a validade dos valores obtidos a partir da técnica.

A influência do comportamento da luz natural na compilação das imagens de calibração, conforme observado em trabalho anterior (SCARAZZATO; SOUZA, 2011), demonstra a necessidade de um enfoque diferenciado para o estabelecimento de uma curva de resposta, para fins de estudo detalhado das condições de luz. Contudo, para o usuário final, que utilizará as imagens como fonte de luz e análise menos detalhada, uma abordagem simplificada demonstra ser suficiente.

\subsubsection{Obtenção das imagens}

As câmeras, as objetivas e o luxímetro utilizados na medição foram colocados em tripés e apontados para o zênite. Um nível portátil foi acoplado às câmeras para verificar o correto enquadramento da cena. As imagens e medições foram coletadas em localidade situada nas coordenadas $22^{\circ} 57^{\prime} 48,6^{\prime \prime}$ 'Sul e 464 $45^{\prime} 42,6^{\prime \prime}$ Oeste, onde foi possível obter um enquadramento com a menor ocorrência possível de obstrução. Essa abordagem se fez necessária para efetuar o registro completo da abóbada celeste, aproveitando-se ao máximo o ângulo de visão dos equipamentos e minimizando-se os possíveis erros de cálculo.

\subsection{Extração dos dados}

Após a tomada das fotografias e a obtenção dos dados de calibração, as imagens foram compiladas no programa Photosphere 1.8.7u (WARD, 2012) e armazenadas no formato Radiance RGBE (extensão .hdr). De posse das imagens, foi elaborada uma rotina em MATLAB para leitura, recorte e extração dos valores de luminância com o auxílio da biblioteca HDR Toolbox,compilada por Banterle et al. (2011). O algoritmo está dividido nas seguintes etapas:

1. Manipulação da imagem e coleta das luminâncias.
2. Construção de modelos de subdivisão do céu.

3. Cálculo dos valores de iluminância.

\subsubsection{Coleta das luminâncias}

Nesta etapa do algoritmo, realiza-se primeiramente a leitura da imagem HDR com o uso da rotina hdrread() do HDR Toolbox, que transforma a imagem de tamanho $\mathrm{MxN}$ no padrão RGBE, com quatro canais de números inteiros, em uma matriz computacional de mesmo tamanho, com três canais de números em ponto flutuante com precisão simples. Efetua-se também a leitura do cabeçalho da imagem para coleta do valor de exposição, variável necessária para o cálculo da matriz de luminância, segundo a fórmula:

$$
L=179 \frac{(0,265 R+0,670 G+0,065 B)}{E}(\text { MISCHLER, 2001) }
$$

em que $\mathrm{L}$ é a luminância em $\mathrm{cd} / \mathrm{m}^{2}$, $\mathrm{E}$ é o valor de exposição da HDR, R o valor do pixel no canal 1 da matriz, G o valor do canal 2 e B o valor do canal 3, representando, respectivamente, as cores vermelha, verde e azul. O resultado da fórmula é uma matriz MxN com apenas um canal de números reais. Após o cálculo da luminância, um recorte da imagem é realizado para obter apenas a hemisfera registrada na fotografia. Para isso, a matriz $\mathrm{MxN}$ original é recortada e rotacionada em torno do eixo vertical. Tal processo produz uma matriz quadrada $\mathrm{NxN}$, sendo $\mathrm{N}$ um valor ímpar igual a $2 r+1$, em que r é o raio da hemisfera da imagem circular produzida pela objetiva olho-depeixe. Nessa etapa de desenvolvimento, os valores de $x$, y e $r$ foram obtidos manualmente, mas é desejável a possibilidade de automatizar a detecção da figura circular na imagem.

\subsubsection{Construção dos modelos}

Os valores de $\mathrm{N}$ e $\mathrm{r}$ foram usados para criar as matrizes de altura angular e azimute (Figura 2), utilizando-se as fórmulas de construção descritas por Snyder (1987) e convertendo-se as coordenadas espaciais $(x, y)$ de cada pixel em coordenadas angulares, tendo por centro o ponto ( $\mathrm{N}-\mathrm{r}, \mathrm{N}-\mathrm{r})$ da matriz. Neste artigo, foram usadas as fórmulas referentes à projeção equisólida.

As matrizes são utilizadas para efetuar a divisão do céu em subzonas pré-determinadas, criando uma matriz NxN,em que o valor de cada subzona é etiquetado no pixel correspondente. A seguir, o zoneamento é aplicado sobre a matriz de luminância, extraindo-se os valores de $L$ de cada fração do céu subdividido e calculando-se sua média aritmética. Nesta pesquisa, foram utilizados dois grupos de 
Figura 2: Matriz de altura angular ( 0 a $\pi / 2$ radianos) e azimute ( 0 a $2 \pi$ radianos)

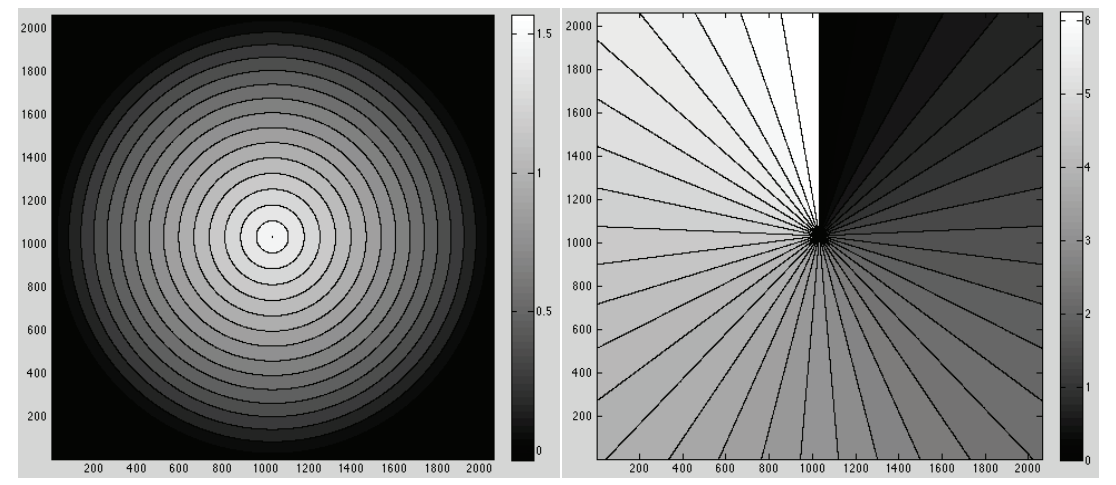

subdivisões de céu, montados sobre a matriz de tamanho $\mathrm{NxN}$, que foram comparados à distribuição pixel a pixel da matriz pura (Figura 3):

1. Modelos pré-definidos usados na construção de equipamentos de medição (TREGENZA, 1987) e em software de simulação de iluminação (CABÚS, 2002), conforme as Figuras 4 e 5.

2. Modelos hipotéticos, representando diferentes subdivisões em 1, 2, 4, 7, 12, 22, 37, 62 e

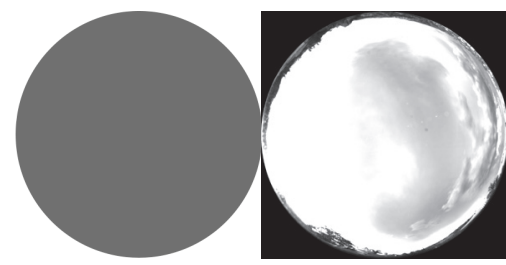

Figura 3: Matriz original

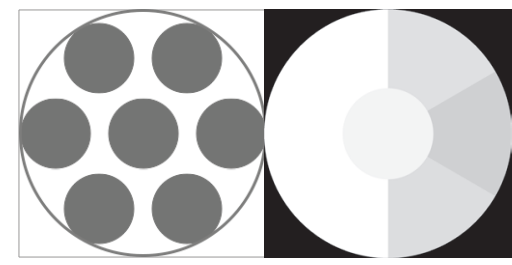

Figura 6: 1 faixa(7 divisões)

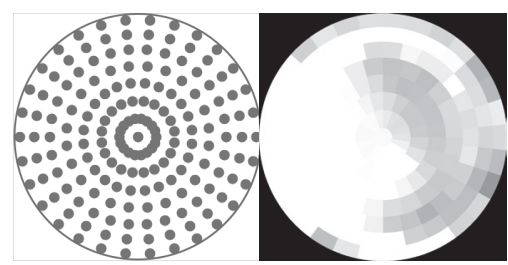

Figura 9: 7 faixas (177 divisões)

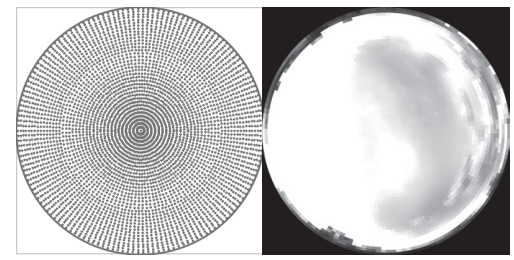

Figura 12: 37 faixas (4581 divisões)

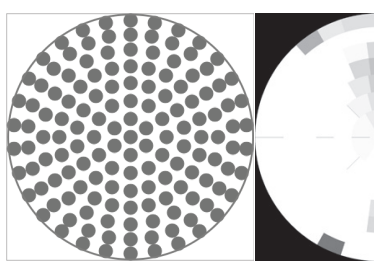

Figura 4: Tregenza (1987)(145 divisões)

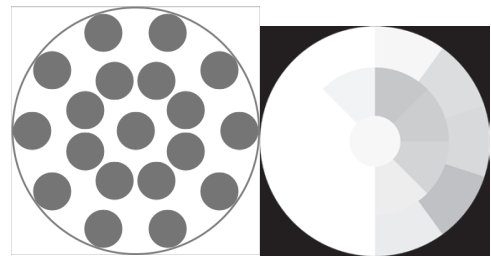

Figura 7: 2 faixas(19 divisões)

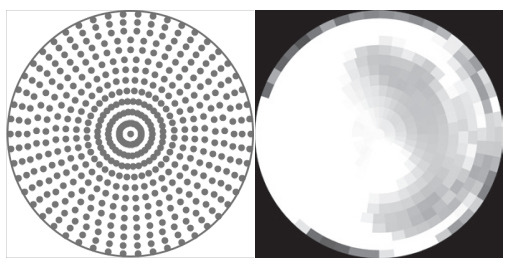

Figura 10: 12 faixas (489 divisões)

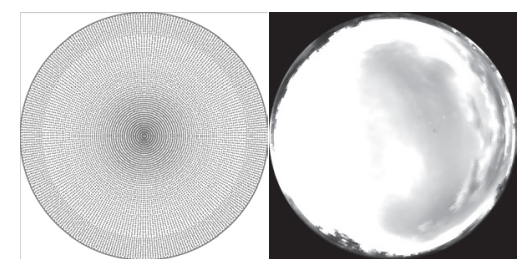

Figura 13: 62 faixas (13051 divisões)

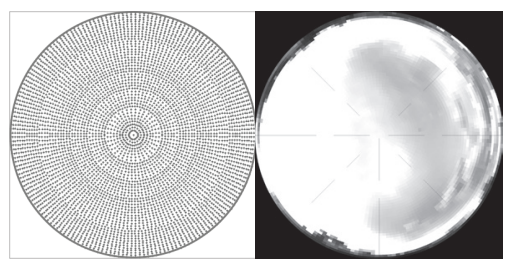

Figura 5: Cabús(2003) (5221 divisões)

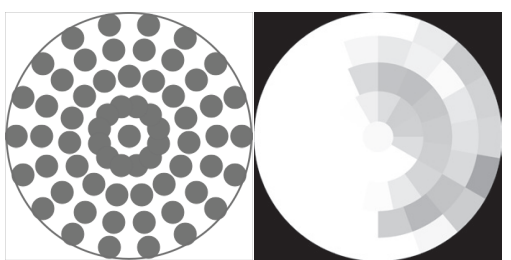

Figura 8: 4 faixas (64 divisões)

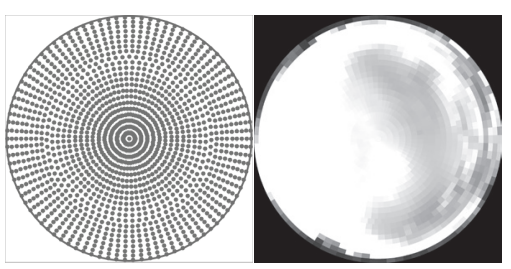

Figura 11: 22 faixas (1628 divisões)

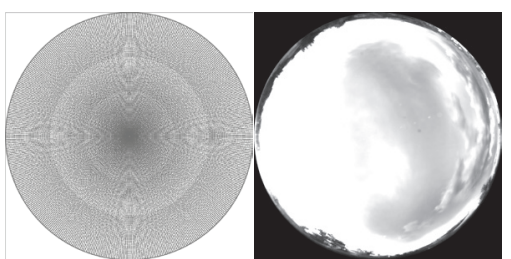

Figura 14: 112 faixas (42151 divisões) 


$$
\Delta E=\sum D_{\theta} L_{\theta} \Delta S_{\theta}
$$

em que $L_{\theta}$ é a luminância, $\Delta S_{\theta}$, o ângulo sólido e $D_{\theta}$, o coeficiente de contribuição de cada ponto do céu. Estando o ponto horizontal em um campo desobstruído, o coeficiente é igual ao seno do ângulo de incidência $\theta$. Assim, a fórmula acima foi usada para calcular a iluminância prevista da HDR, sendo o ângulo sólido calculado a partir da divisão do valor total de $2 \pi$, valor da área da superfície de uma hemisfera, pela quantidade de pontos válidos, algo possível por se tratar de uma projeção que preserva as áreas dos objetos.

\section{Análise de resultados}

A seguir, apresentam-se os resultados obtidos nesta pesquisa. No total, foram comparadas 190 imagens HDR do céu, sendo 108 obtidas com a câmera Canon EOS (Figura 15) e $82 \mathrm{com}$ a Nikon D60 (Figura 16). As imagens foram capturadas num intervalo de tempo de duas semanas, em Fevereiro de 2012.

\subsection{Calibração}

Os resultados da calibração estão descritos na Tabela 2 e nas Figuras17 e 18. Os números obtidos mostram uma regularidade no comportamento das câmeras, que se reflete também na similaridade das curvas obtidas. Tais características sugerem que as câmeras podem ter um comportamento similar nas demais etapas.

\subsection{Comparação dos dados}

As Figuras 19 e 20 mostram a comparação entre as iluminâncias obtidas a partir das imagens HDR das duas câmeras e as do luxímetro T-10A.
As duas câmeras tiveram comportamento análogo nas comparações. Parte dos céus registrados apresentaram resultados muito próximos das medições do luxímetro. Tratava-se de céus claros ou encobertos, com pouca ou nenhuma influência da movimentação das nuvens na distribuição da luz na abóbada celeste. Os céus com maior discrepância entre as imagens e o equipamento de medição eram céus parcialmente encobertos, com alto dinamismo na formação e deslocamento das nuvens.

As Figuras 21 a 28 mostram a comparação entre as iluminâncias obtidas a partir das imagens HDR e as diferentes subdivisões hipotéticas aplicadas.

Tabela 2: Características dos materiais utilizados na simulação computacional.

\begin{tabular}{|c|c|c|c|c|c|}
\hline PONTO & $\begin{array}{c}\mathrm{LS}-110 \\
\left(\mathrm{CD} / \mathrm{M}^{2}\right)\end{array}$ & $\begin{array}{c}\mathrm{D} 60 \\
\left(\mathrm{CD} / \mathrm{M}^{2}\right)\end{array}$ & $\begin{array}{c}\mathrm{XSI} \\
\left(\mathrm{CD} / \mathrm{M}^{2}\right)\end{array}$ & $\begin{array}{c}\mathrm{FC} \\
(\mathrm{LS} / \mathrm{D} 60)\end{array}$ & $\begin{array}{c}\mathrm{FC} \\
(\mathrm{LS} / \mathrm{XS})\end{array}$ \\
\hline $\mathbf{1}$ & $\mathbf{3 5 2 0}$ & $\mathbf{2 8 5 0}$ & $\mathbf{3 1 9 0}$ & $\mathbf{1 , 2 4}$ & $\mathbf{1 , 1 0}$ \\
\hline 2 & $\mathbf{7 1 0 0}$ & $\mathbf{6 3 9 0}$ & $\mathbf{6 9 3 0}$ & $\mathbf{1 , 1 1}$ & $\mathbf{1 , 0 2}$ \\
\hline 3 & $\mathbf{3 6 9 0}$ & $\mathbf{3 1 9 0}$ & $\mathbf{3 2 2 0}$ & $\mathbf{1 , 1 6}$ & $\mathbf{1 , 1 5}$ \\
\hline 4 & $\mathbf{7 5 3 0}$ & $\mathbf{7 0 1 0}$ & $\mathbf{7 2 9 0}$ & $\mathbf{1 , 0 7}$ & $\mathbf{1 , 0 3}$ \\
\hline $\mathbf{5}$ & $\mathbf{4 3 0}$ & $\mathbf{3 5 0}$ & $\mathbf{3 2 0}$ & $\mathbf{1 , 2 3}$ & $\mathbf{1 , 3 4}$ \\
\hline $\mathbf{6}$ & $\mathbf{3 5 8 0}$ & $\mathbf{3 3 8 0}$ & $\mathbf{3 4 4 0}$ & $\mathbf{1 , 0 6}$ & $\mathbf{1 , 0 4}$ \\
\hline 7 & $\mathbf{4 6 0}$ & $\mathbf{3 8 0}$ & $\mathbf{3 8 0}$ & $\mathbf{1 , 2 1}$ & $\mathbf{1 , 2 1}$ \\
\hline $\mathbf{8}$ & $\mathbf{3 2 3 0}$ & $\mathbf{3 0 6 0}$ & $\mathbf{3 2 2 0}$ & $\mathbf{1 , 0 6}$ & $\mathbf{1 , 0 0}$ \\
\hline $\mathbf{9}$ & $\mathbf{4 3 0}$ & $\mathbf{3 3 0}$ & $\mathbf{3 6 0}$ & $\mathbf{1 , 3 0}$ & $\mathbf{1 , 1 9}$ \\
\hline $\mathbf{1 0}$ & $\mathbf{6 4 0 0}$ & $\mathbf{5 9 7 0}$ & $\mathbf{6 3 1 0}$ & $\mathbf{1 , 0 7}$ & $\mathbf{1 , 0 1}$ \\
\hline $\mathbf{1 1}$ & $\mathbf{3 8 0 0}$ & $\mathbf{3 0 9 0}$ & $\mathbf{3 2 2 0}$ & $\mathbf{1 , 2 3}$ & $\mathbf{1 , 1 8}$ \\
\hline & & & MÉDIA FC & 1,16 & 1,12 \\
\hline
\end{tabular}

As menores subdivisões foram tratadas como hipótese, pois era esperado que a grande abstração do céu produzida por esses modelos levasse a grandes erros de cálculo. A média para a divisão de 1 faixa foi de $7,56 \%$ para a Canon EOS e $9,82 \%$ para a Nikon D60, enquanto que para a de 2 faixas foi de $2,13 \%$ e $2,83 \%$, respectivamente.

As subdivisões de 4 e 7 faixas foram mais estáveis,

Figura 15: Imagens obtidas com a Canon EOS

Figura 16: Imagens obtidas com a Nikon D60

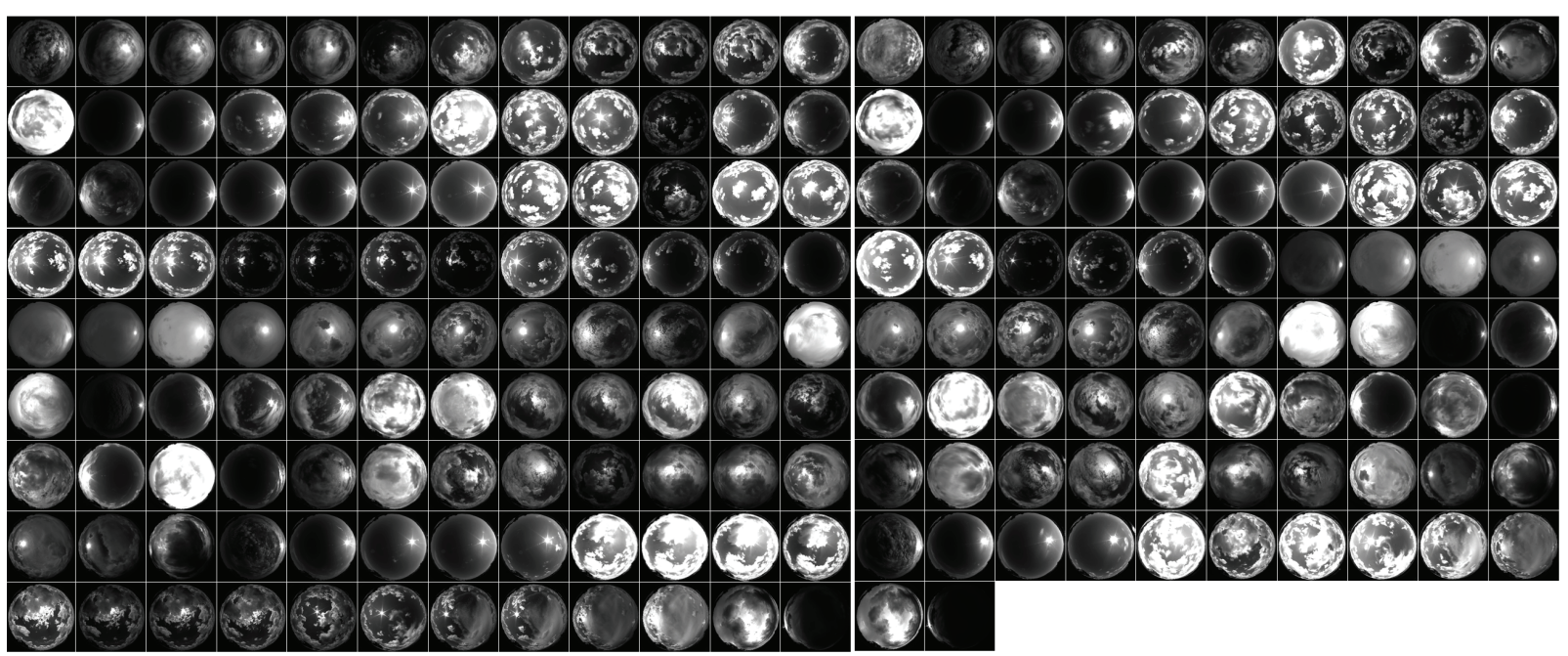


Figura 17: Curva de resposta da Nikon D60

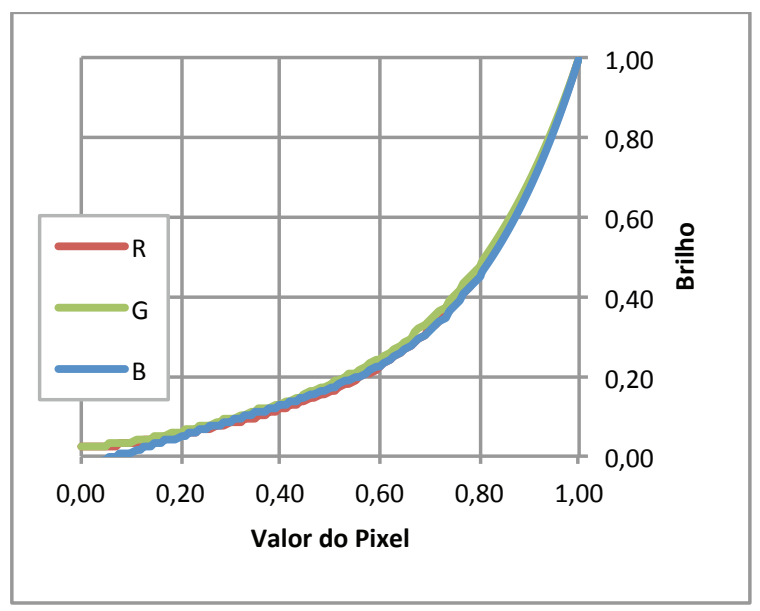

Figura 19: Comparação entre Canon EOS e Minolta T-10A
Figura 18: Curva de resposta da Canon EOS

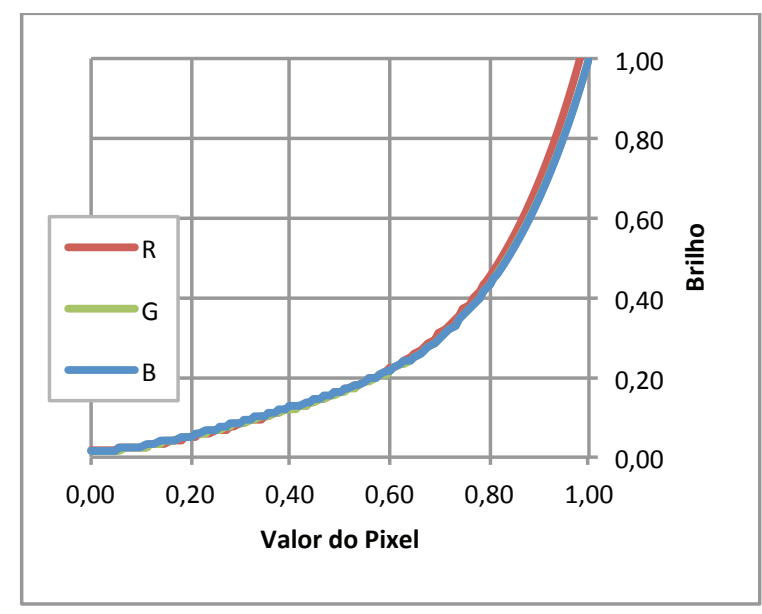

Figura 20: Comparação entre Nikon D60 e Minolta T-10A

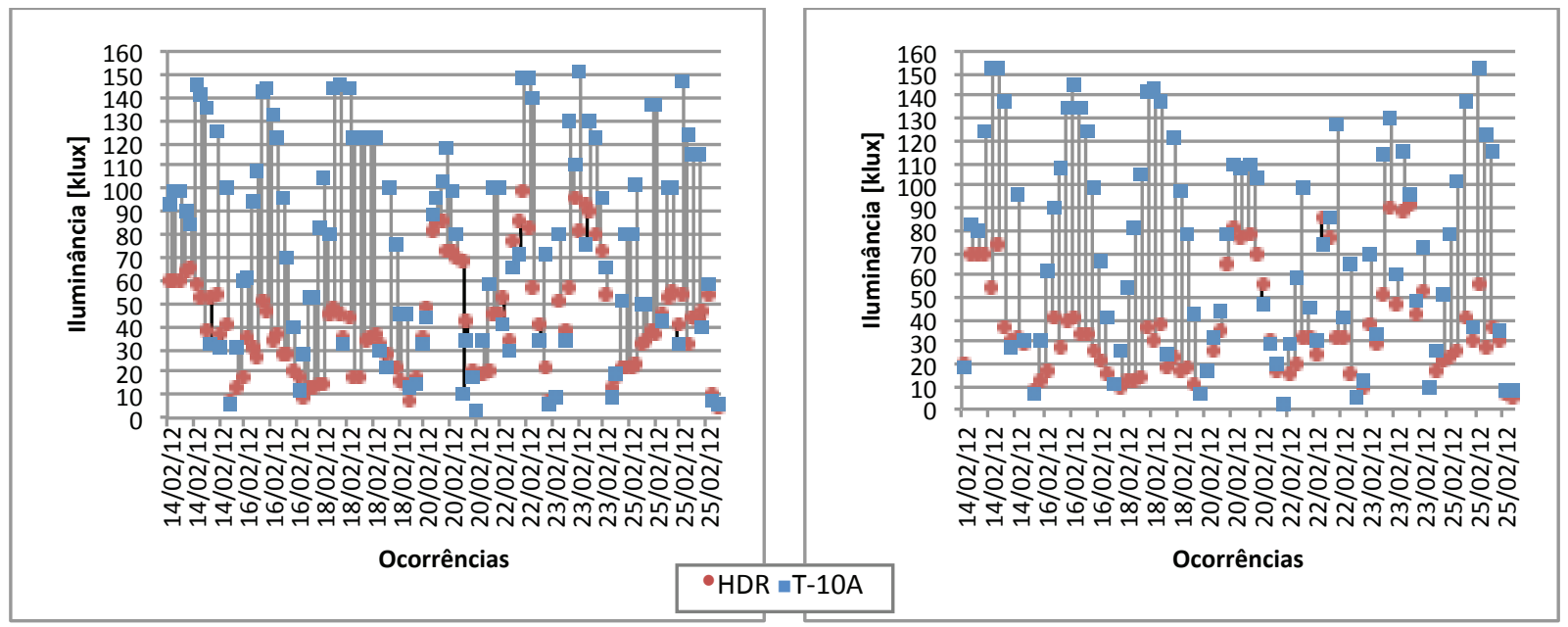

apesar de alguns picos com erros superiores a $2 \%$, para mais e para menos. A média para a divisão de 4 faixas foi de $0,87 \%$ para a Canon EOS e $0,81 \%$ para a Nikon D60, enquanto que para a de 7 faixas foi de $0,31 \%$ e $0,47 \%$, respectivamente.

As subdivisões de 12 e 22 faixas, por sua vez, demonstraram erros mínimos, inferiores a $0,5 \%$, para mais e para menos. Apenas em um caso o erro chegou a $1,53 \%$. A média para a divisão de 12 faixas foi de $0,085 \%$ para a Canon EOS e $0,083 \%$ para a Nikon D60, enquanto que para a de 22 faixas foi de $0,022 \%$ e $0,017 \%$, respectivamente.

Por fim, as maiores subdivisões apresentaram os resultados mais próximos aos obtidos com o arquivo original. A média para a divisão de 37 faixas foi de $0,0069 \%$ para a Canon EOS e $0,0063 \%$ para a Nikon D60; para a de 62 faixas, a média foi de $0,028 \%$ e $0,0019 \%$, enquanto que, para a de 112 faixas, foi de $0,0007 \%$ e $0,0008 \%$, respectivamente.É perceptível que o número de divisões influencia na quantidade de erros e no distanciamento entre o modelo e a medição pixel a pixel, como era esperado. Os dados acima corroboram com os obtidos a partir da divisão clássica proposta por Tregenza (1987), e da variação para céus claros utilizada por Cabús (2003), que tiveram comportamentos similares às divisões hipotéticas de 7 faixas e 37 faixas, respectivamente, como mostram as Figuras 29 e 30.

Deve ser ponderado, nessa relação, o custo computacional para a realização da tarefa de divisão da imagem em cada subdivisão. As Figuras $31 \mathrm{e}$ 32 mostram o tempo de cálculo para a criação de cada modelo hipotéticosobre a imagem HDR, em comparação com a média do absoluto das diferenças encontradas em cada caso. Conforme esperado, a progressão dos modelosresultana diminuição da quantidade de erros e no aumento do tempo de cálculo. Coincidentemente, as duas curvas se aproximam de exponenciais e são inversamente proporcionais.

\section{Conclusões}

A partir dos resultados obtidos é possível constataro comportamento similar das câmeras nos processos. 
Figura 21: Distribuição dos erros para Canon EOS

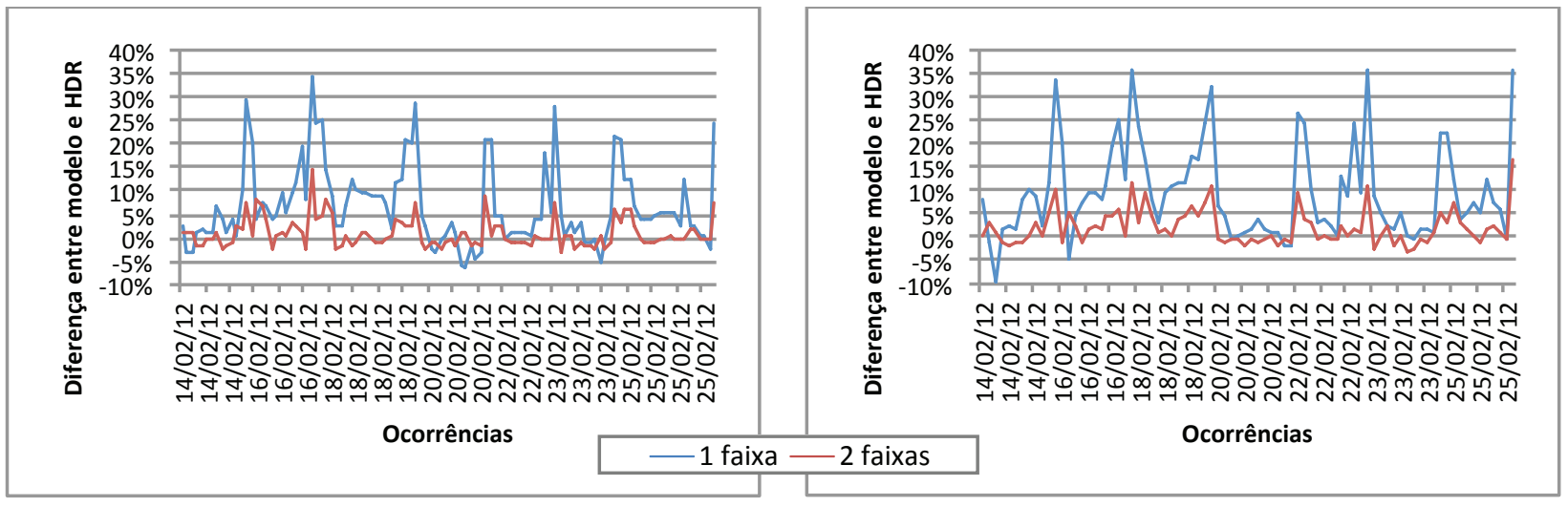

Figura 23: Distribuição dos erros para Canon EOS

Figura 24: Distribuição dos erros para Nikon D60

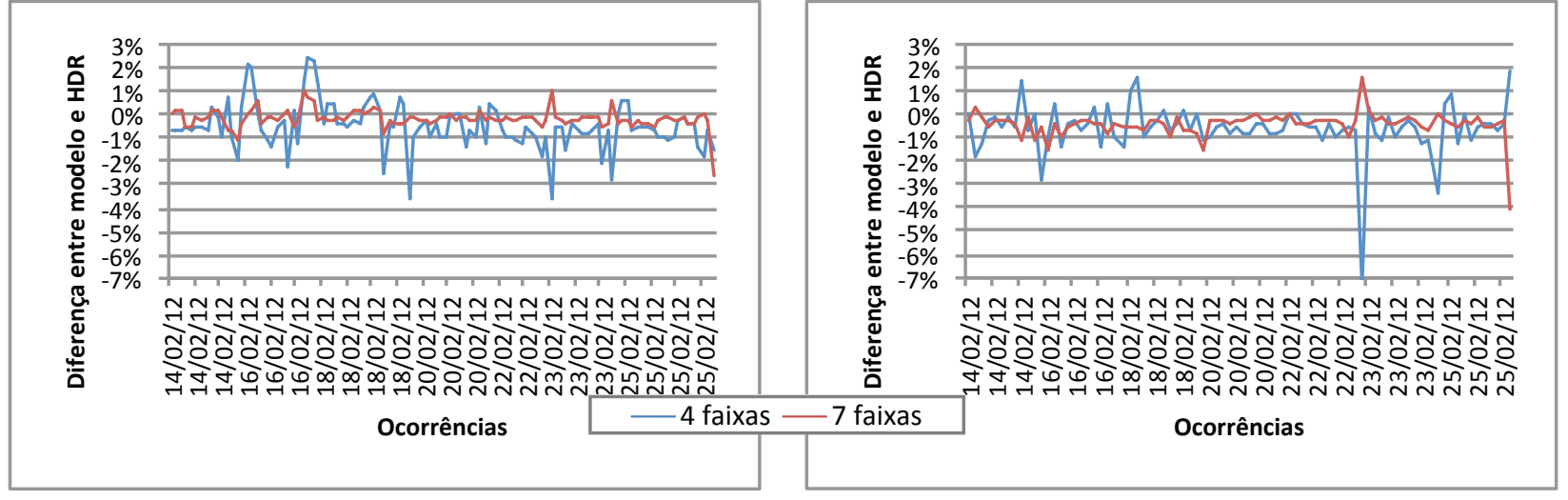

Figura 25: Distribuição dos erros para Canon EOS

Figura 26: Distribuição dos erros para Nikon D60

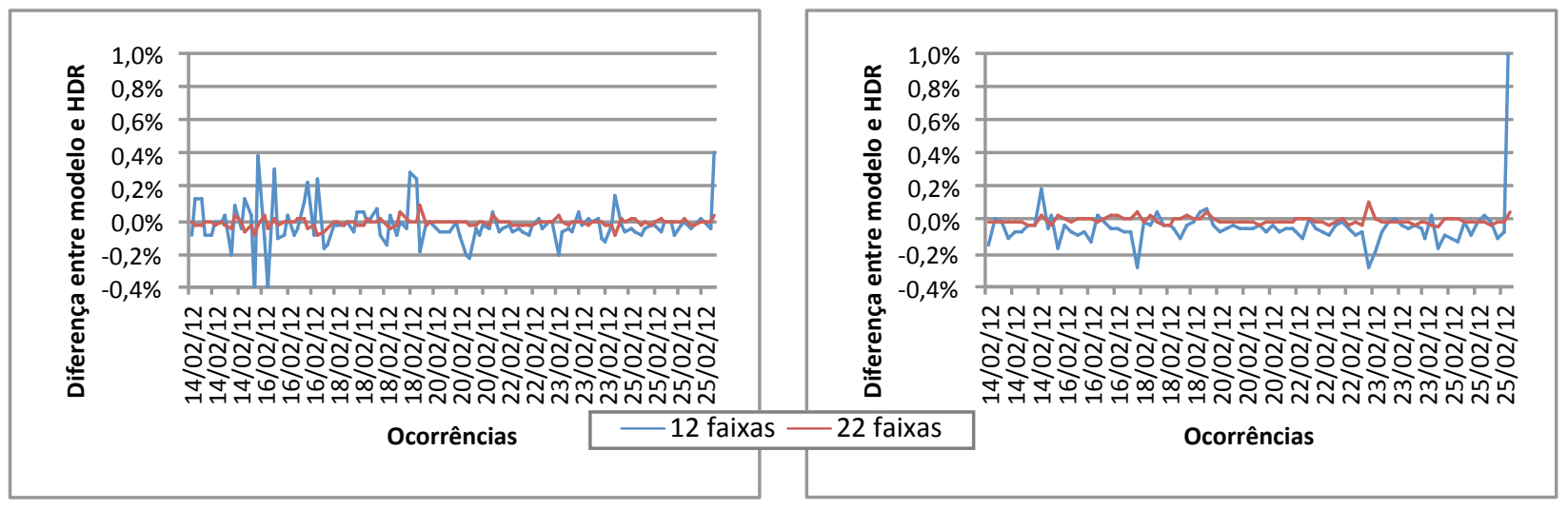

Figura 27: Distribuição dos erros para Canon EOS

Figura 28: Distribuição dos erros para Canon EOS

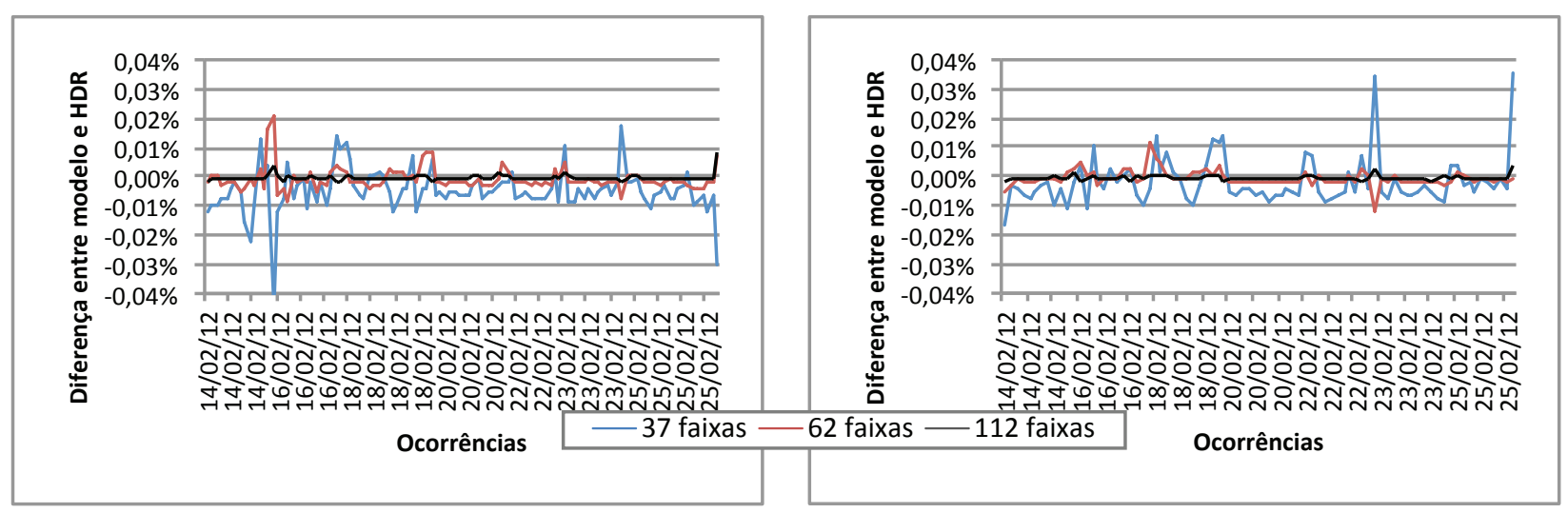




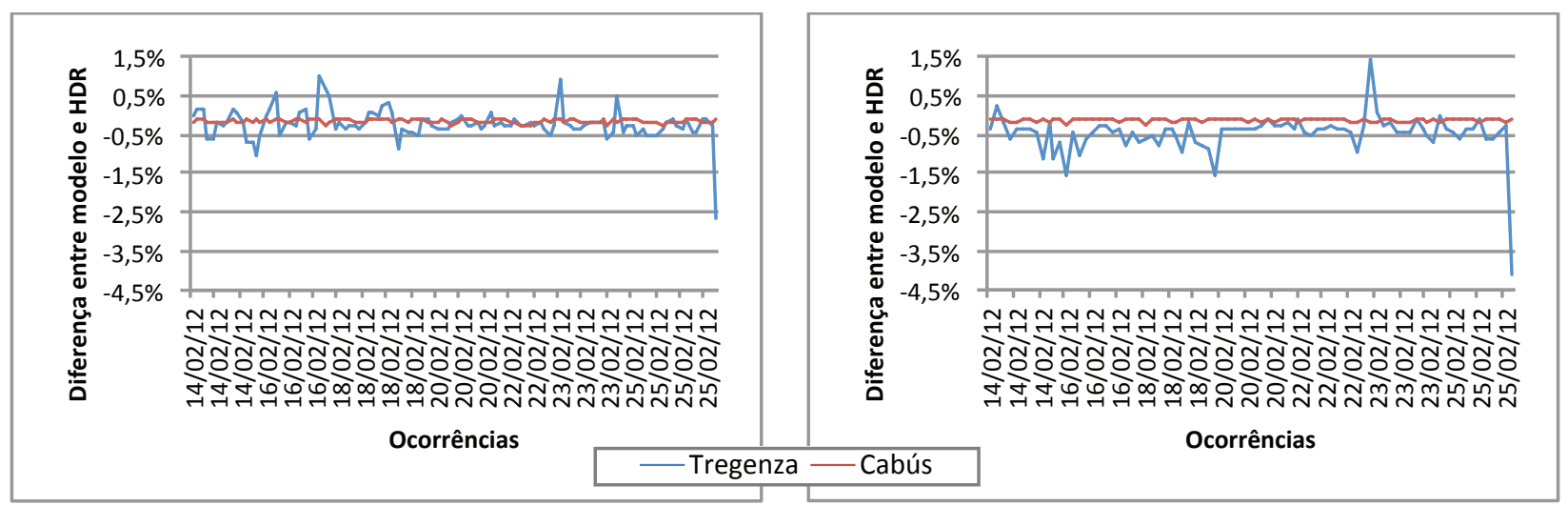

Figura 31: Média de erro e tempo de cálculo para Canon EOS

Figura 32: Média de erro e tempo de cálculo para Nikon D60

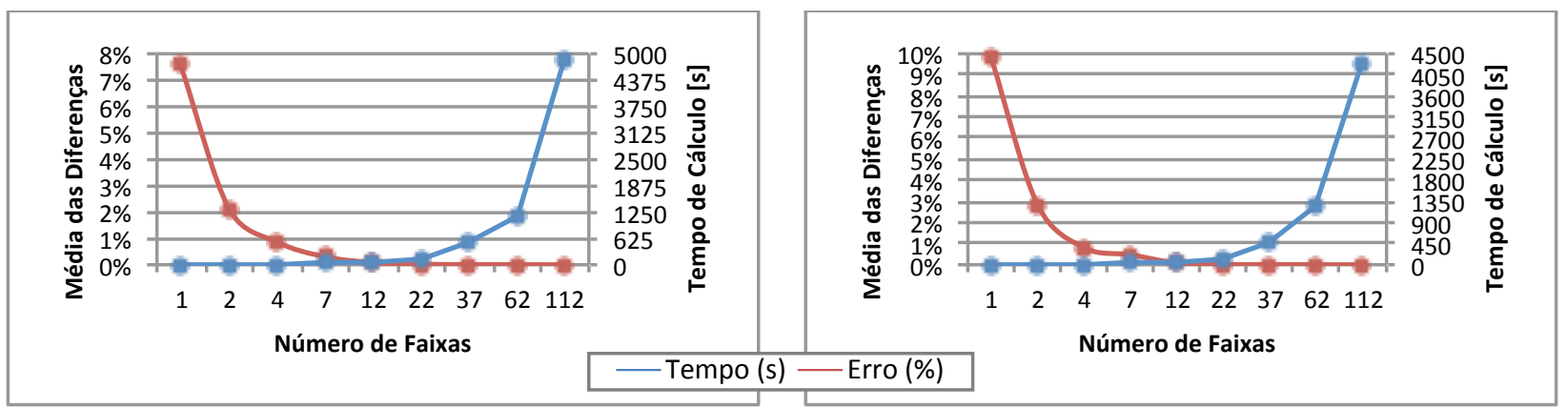

Isso é importante para a verificação da confiabilidade da imagem HDR como instrumento de medição, independentede marca ou modelo específico do equipamento fotográfico.

Quanto à comparação com o luxímetro, as diferenças podem ser atribuídas à precisão inerente ao processo de medição com o equipamento portátil. Afinal, valores instantâneos de iluminância estão sujeitos a enormes variações, dado o dinamismo do céu. Assim, as maiores diferenças estão nos padrões de céu que se aproximam do parcialmente nublado, que são os mais dinâmicos que existem. Nesse caso, a diferença de alguns segundos entre as medições dos equipamentos exerce grande influência nos resultados obtidos e, logo, nas diferenças observadas. Cabe observar também que a tomada das fotografias não é instantânea, demorando algo em torno de dez a quinze segundos.Com vistas à obtenção de resultados comparativos mais precisos, dados de uma estação medidorade iluminação natural obtidos em conjunto com a fotografia HDR podem ajudar na verificação dos resultados.

É patente que os modelos de divisão do céu exercem influência considerável no resultado dos cálculos. Céus de baixa resolução tendem a ter cálculos rápidos, mas maiores discrepâncias nos resultados. Maiores resoluções, por sua vez, levam a resultados mais precisos, mas com grande acréscimo na variável tempo de cálculo. As resoluções médias (em torno de 500 a 1000 divisões) possuem resultados satisfatórios a um baixo custo computacional. Assim como nos modelos virtuais, a relação entre tempo e precisão deve ser considerada de acordo com as necessidades do usuário.

Por fim, deve-se ressaltar que a precisão obtida com a maioria dos modelos aplicados permite considerar que há a possibilidade de aplicação de valores reais de imagens HDR como fonte de luz em programas de simulação virtual para cálculos de iluminação natural.

\section{Agradecimentos}

O presente trabalho contou com o apoio da Fundação de Amparo à Pesquisa do Estado de São Paulo (FAPESP), através dos processos 2011/10314-7 e 2011/22065-1. As opiniões, hipóteses e conclusões ou recomendações expressas neste material são de responsabilidade do(s) autor(es) e não necessariamente refletem a visão da FAPESP.

\section{Referências Bibliográficas}

BANTERLE, F.; ARTUSI, A.; DEBATTISTA, K.; CHALMERS, A.Advanced High Dynamic Range Imaging: Theory and Practice. 1. ed. [S.I.] A K Peters/CRC Press, 2011. 
CABÚS, R. Tropical daylighting: predicting sky types and interior illuminance in north-east Brazil. Sheffield, Reino Unido: School of Architectural Studies, 2002.

CHAMAIDI, T. Calibrated Sky Luminance Maps for Daylight Simulation, 19. jun. 2006. Master of Science, Vienna, Áustria: Vienna University of Technology - TU Vienna.

CHENEY, K.; INANICI, M. Image Based Rendering: Using High Dynamic Range Photographs to Light Architectural Scenes Architecture in the age of digital reproduction. Anais... In: 2008 ACSA FALL CONFERENCE. Illinois, EUA: out. 2008

INANICl, M. Applications of Image based Rendering in Lighting Simulation: Development and evaluation of Image based Sky Models Building Simulation 2009. Anais... In: 11TH INTERNATIONAL BUILDING PERFORMANCE SIMULATION ASSOCIATION (IBPSA) CONFERENCE. Glasgow, Reino Unido: jul. 2009.

INANICI, M. N. Per-pixel data analysis. Berkeley: Ernest Orlando Lawrence Berkeley National Laboratory, 2005. Disponível em <http://dmg.caup. washington.edu/mehlika/mi-CIE05.pdf> Acesso em: 19 set. 2005

INANICI, M. N.; GALVIN, J. Evaluation of High Dynamic Range photography as a luminance mapping technique. Berkeley: Lawrence Berkeley National Laboratory, 2004. Disponível em: <http://www.osti.gov/bridge/ servlets/purl/841925-QBBn0i/native/841925.pdf> e em: <http://repositories.cdlib.org/lbnl/LBNL-57545>. Acesso em: 04 mar. 2009.

JACOBS, A. High Dynamic Range Imaging and its Application in Building Research. Advances in Building Energy Research, v. 1, n. 1, p. 177-202, 2007.

MISCHLER, Georg. Mensagem postada em lista de discussão, 2001. Disponível em: <http://www.radianceonline.org/pipermail/radiance-general/2001December/000097.html> Acesso em: 06/10/2011.

PEREIRA, R. C. Avaliação de ferramentas de simulação de iluminação natural por meio de mapeamento digital de luminâncias da abóboda celeste e entorno, 25.nov. 2008. Doutorado, Florianópolis, SC: Universidade Federal de Santa Catarina - UFSC.

REINHARD, E.; WARD, G.; PATTANAIK, S.; DEBEVEC, P.High dynamic range imaging: acquisition, display, and image-based lighting. San Francisco (CA): Elsevier; Morgan Kaufmann Publisher, 2006.
ROY, G.; HAYMAN, S.; JULIAN, W. Sky analysis from CCD images: cloud cover. Lighting Research and Technology, v. 33, n. 4, p. 211-221, 12 jan. 2001.

SCARAZZATO, P.; SOUZA, D. F. HDR Images in the Study of Sky Vault Luminance Distribution and Identification of prevailing Sky Types. Sun City, África do Sul: 27th Session of the CIE. Proceedings..., 2011. v. 1. p. $758-766$.

STUMPFEL, J.; TCHOU, C.; JONES, A.; HAWKINS, T.; WENGER, A.; DEBEVEC, P. Direct HDR capture of the sun and sky Proceedings of the 3rd international conference on Computer graphics, virtual reality, visualisation and interaction in Africa. Anais...: AFRIGRAPH '04.New York, NY, USA: ACM, 2004 Disponível em: <http://doi.acm. org/10.1145/1029949.1029977>

SNYDER, J. P. Map projections--a working manual. [S.I.] U.S. G.P.O., 1987.

TREGENZA, P. R. Subdivision of the sky hemisphere for luminance measurements. Lighting Research and Technology, v. 19, n. 1, p. $13-14,1$ mar. 1987.

TREGENZA, P. R.; WATERS, I. M. Daylight coefficients. Lighting Research and Technology, $v$. 15, n. 2, p. $65-71,1$ jun. 1983.

WARD, G. Photosphere 1.8.7u.2012, Disponível em: <http://anyhere.com/> Acesso em: 25/06/2012. 
SOUZA, D. F.; SCARAZZATO, P. S.; PEDRINI, H.. Influência da divisão do céu em cálculos de disponibilidade de luz a partir de imagens de grande alcance dinâmico. Paranoá, Brasília, n 12, p. 105-114, 2014. 